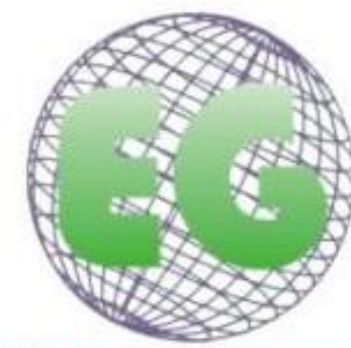

ISSN 1695-6141 $N^{\circ} 50$

\title{
El cuidado informal en tiempos de crisis. Análisis desde la perspectiva enfermera
}

Informal care in times of crisis. Analysis from the nursing perspective

Manoli Cantillo ${ }^{1}$

Teresa Lleopart $^{2}$

Sandra Ezquerra ${ }^{3}$

${ }^{1}$ Diplomada en Enfermería, Máster en Envejecimiento Activo y Saludable, Máster en Atención y Cuidados paliativos. Universitat de Vic-Universitat Central de Catalunya. España.

${ }^{2}$ Diplomada en Enfermería, Licenciada en Antropología Social y Cultural, Suficiencia investigadora en Ciencias Sociales y Salud. Universitat de Vic-Universitat Central de Catalunya. España.

${ }^{3}$ Licenciada en Historia Contemporánea y en Antropología Social y Cultural, Máster y Doctora en Sociología, Universitat de Vic-Universitat Central de Catalunya. España.

E-mail: manoli.cantillo@uvic.cat

http://dx.doi.org/10.6018/eglobal.17.2.297211

Recibido: $15 / 06 / 2017$

Aceptado: 21/09/2017

\section{RESUMEN:}

Objetivos: Cuantificar y caracterizar la producción científica enfermera sobre cuidados informales del período 2007-2016, observar la evolución de la temática durante estos años, adquirir una perspectiva actual sobre el estado de la cuestión y realizar propuestas sobre futuras líneas de investigación e intervención.

Metodología: Revisión bibliográfica llevada a cabo mediante dos estrategias: una cuantitativa, y una segunda estrategia cualitativa.

Resultados: El tipo de artículo más publicado es el estudio original cuantitativo, aunque se detecta un crecimiento de las publicaciones con enfoque cualitativo. Los temas más tratados son el perfil de la persona cuidadora, los impactos de la atención en su salud y en otros aspectos de su vida cotidiana, las propuestas de intervenciones profesionales para promover el cuidado personal y para evitar la sobrecarga de las personas cuidadoras y, por último, el uso de herramientas de evaluación para la planificación de la atención a las mismas.

Conclusiones: Las publicaciones enfermeras identifican con acierto la centralidad del cuidado informal y el giro asistencial hacia el domicilio y la familia. No problematizan, sin embargo, el actual trasvase de responsabilidades hacia el cuidado desde las administraciones públicas hacia el ámbito familiar, ni analizan en profundidad las desigualdades socioeconómicas y de género reinantes en el actual escenario de cuidados. El abordaje a estos dos elementos puede contribuir a abrir nuevas líneas de investigación e intervención en el campo de la enfermería.

Palabras clave: Cuidado informal; cronicidad; crisis económica; crisis de los cuidados. 


\begin{abstract}
:
Goals: To quantify and characterize the scientific production in nursing on informal care from 2007 to 2016, to observe the evolution of the theme during this period, to acquire a current perspective on the state of the arts, and to suggest future directions of both research and professional practice.

Methods: Bibliographical review undertaken through two strategies: a quantitative strategy and a qualitative one.

Results: The most frequent type of published article is quantitative although there is an increase of qualitative publications. Among the most frequent themes are: the study of the caregiver's profile, as well as the impacts of care on their health and on their everyday life; practical professional recommendations to promote care and self-care and to prevent caregivers' overload; and, finally, the use of assessment tools for planning attention of caregivers.

Conclusions: While nursing publications rightly identify the centrality of the family and the household in the new care scenario, they do not problematize the current transfer of responsibility for care from public administrations toward the realm of the family. Neither do they problematize the social, economic, and gender inequalities that take place in the context of care. To approach these two themes can contribute to create new research and professional lines in nursing.
\end{abstract}

Keywords: Informal care; Chronicity; Economic crisis; Caregiving crisis.

\title{
INTRODUCCIÓN
}

En los últimos años se ha dado una creciente presencia de análisis de los cuidados informales de larga duración en áreas de conocimiento diversas, tanto en el campo de las ciencias de la salud como en el de las ciencias sociales, pero no se ha realizado aún un abordaje sistemático de la presencia e importancia de esta cuestión en el ámbito de la enfermería.

Los cuidados informales se definen como la prestación de cuidados a personas en situación de dependencia por parte de la familia, basados en relaciones afectivas, siendo tarea producida en el hogar con un rol adscrito a la mujer ${ }^{(1)}$. Éstos suelen ser llevados a cabo en el marco del hogar, casi el $90 \%$ del tiempo total de cuidado recibido por las personas mayores recae sobre la familia ${ }^{(2)}$, y son las mujeres quienes asumen la mayor parte ${ }^{(3-5)}$.

La provisión de cuidados comporta una dedicación significativa de energía y tiempo para la persona cuidadora. El 95,9\% de las personas cuidadoras de personas de 650 más años de edad declara prestar cuidados de 6 a 7 días a la semana y el 38,9\% dedica al menos 16 horas diarias a esta labor ${ }^{(4)}$. Todo ello tiene un impacto negativo en la salud y en las relaciones familiares, profesionales y sociales de las personas cuidadoras, así como en su calidad de vida ${ }^{(4,6)}$.

Los cambios socio demográficos y culturales de las últimas décadas, la variación del rol de las mujeres aumentando su presencia en el mercado laboral y el cambio de modelo familiar, introdujeron demandas y retos al Estado del Bienestar ${ }^{(7)}$. En el 2006 con la Ley de la Promoción de la Autonomía Personal y Atención a las Personas en Situación de Dependencia (LAPAD) en España, el Estado adoptaba una regulación que unificaba el marco básico de derechos individuales y subjetivos de las personas en situación de dependencia funcional mediante la creación de una cartera de

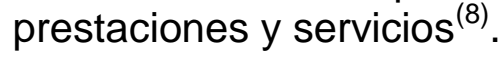

Debido a la crisis económica iniciada en el 2008 todas las partidas presupuestarias estatales llevaron consigo apareada la palabra "recortes" y todo avance obtenido en este sentido se pierde. Diez años después de su nacimiento se evidencia el fracaso de la pretensión de la Ley, cuestionando de nuevo qué respuestas se deberán dar a 
los cuidados informales y la atención a la dependencia. Como resultado tanto de los recortes en el sistema de bienestar como de las crecientes situaciones de cronicidad, el sistema de salud en los próximos años tendrá como reto la atención de las personas con problemas de dependencia, pero de manera diferente a la existente hasta el momento.

Se plantea un nuevo modelo organizativo que aspira a ser accesible y capaz de proporcionar respuestas adecuadas a las necesidades emergentes ${ }^{(9)}$. El diseño de este nuevo modelo trabaja con la doble premisa de que el domicilio es el mejor lugar donde los pacientes crónicos pueden mantener el control de su cuidado ${ }^{(10)}$ y, que la salud comunitaria debe ser integrada al sistema asistencial en tanto que constituye el nivel básico de atención que garantiza la globalidad y la continuidad de los cuidados $^{(11,12)}$, en este escenario la enfermera dispone de una visión integral que contextualiza la necesidad y capacidad de auto cuidado ${ }^{(10,13,14)}$.

El nuevo escenario precisará de un posicionamiento y conocimiento por parte de las enfermeras para convertir los retos en oportunidades de impulso, progreso y fortalecimiento de la enfermería comunitaria, ampliando las competencias para que estén centradas en las necesidades del cuidado de pacientes, familias y comunidad e impulsando alianzas más estrechas con otros agentes de cuidado ${ }^{(9)}$. La enfermera deberá reflexionar sobre el nuevo contexto, así como sobre su rol en él.

El objetivo de esta revisión sistemática es identificar las características de la producción científica en el ámbito de la enfermería sobre los cuidados informales del periodo 2007 a 2016 desde la mirada enfermera, vinculando el análisis y las reflexiones realizadas, visibilizando las tendencias presentes y, con el propósito de sugerir futuras líneas de investigación, identificando posibles ausencias en los estudios.

\section{METODOLOGÍA}

Se efectuó una revisión sistemática de publicaciones científicas sobre el cuidado informal y los cuidadores informales en territorio español. La búsqueda se fijó en el período comprendido entre el año 2007 y el 2016 y se realizó a partir de la formulación de las siguientes preguntas: ¿Cuáles son las características y las principales temáticas de las publicaciones en revistas científicas de enfermería sobre los cuidados informales en el período de 2007 a 2016? ¿Reflejan estas publicaciones las alteraciones recientes en el abordaje de la cronicidad y los cuidados informales? ¿Qué aspectos relacionados con el cuidado informal a la cronicidad están recibiendo atención y cuáles no? ¿Cuáles son las temáticas a las que la literatura enfermera presta escasa atención y que, por lo tanto, pueden constituir líneas de investigación fértiles e innovadoras en el futuro?

Para la búsqueda bibliográfica se han utilizado los descriptores "informal care spain", "informal caregivers spain", "family care spain", "care home spain", "family caregivers spain", "care crisis spain", y "economic crisis spain" en las principales bases de datos nacionales e internacionales: Pubmed, Scopus, Dialnet, Cinahl y Scielo. Se han usado, a su vez, los mismos términos en castellano: "cuidados informales", "cuidadores informales", "cuidados familiares", "cuidados en el hogar", "cuidadores familiares", "crisis de los cuidados" y "crisis económica". 
Se incluyeron todos aquellos artículos (originales, de revisión, breves y de reflexión) publicados en castellano o en inglés en revistas de enfermería sobre el cuidado informal y los cuidadores informales de personas mayores en situación de dependencia funcional en un entorno domiciliario en el Estado español.

Se excluyeron los artículos sobre cuidados informales en patologías concretas (demencia, AVC...) o situaciones agudas, los artículos sobre cuidados informales en un contexto hospitalario o de institucionalización y los artículos sobre cuidados en pediatría.

La estrategia de selección consistió en la elaboración de un listado de todos los artículos hallados a partir de la búsqueda por descriptores. A continuación, se procedió a suprimir los artículos duplicados. Finalmente, se realizó una revisión de los títulos y una localización de los resúmenes de los artículos seleccionados, descartando aquellos que no cumplían los criterios de inclusión.

\section{RESULTADOS}

Se obtuvieron 660 artículos de la búsqueda, de los cuales, en una primera lectura de su título y resumen se descartaron 601 por no estar en relación con el objetivo de la investigación.

Finalmente fueron 59 los artículos que cumplían los criterios de inclusión y era accesible el artículo completo.

La relación de los artículos analizados se presenta en la Tabla I.

Tabla I: Relación de artículos analizados.

\begin{tabular}{|c|c|c|c|c|}
\hline Autor/es y año & Artículo & Revista & Metodología & Objetivos \\
\hline $\begin{array}{l}\text { Casado-Mejía, R., \& } \\
\text { Ruiz-Arias, E. (2016) }\end{array}$ & $\begin{array}{l}\text { Factors influencing family } \\
\text { care by immigrant women } \\
\text { in Spain: a qualitative } \\
\text { study }\end{array}$ & $\begin{array}{l}\text { Nursing } \\
\text { Research and } \\
\text { Education }\end{array}$ & $\begin{array}{l}\text { Estudio } \\
\text { cualitativo }\end{array}$ & $\begin{array}{l}\text { Identificar y comprender los factores } \\
\text { que influyen en las relaciones en el } \\
\text { contexto del cuidado informal } \\
\text { prestado por mujeres inmigrantes }\end{array}$ \\
\hline $\begin{array}{l}\text { Peña-lbáñez, F., } \\
\text { Álvarez-Ramírez, M. } \\
\text { A., \& Melero-Martín, J. } \\
(2016)\end{array}$ & $\begin{array}{l}\text { La carga del cuidador en } \\
\text { el cuidado informal de los } \\
\text { pacientes inmovilizados } \\
\text { en una zona básica de } \\
\text { salud urbana }\end{array}$ & $\begin{array}{l}\text { Enfermería } \\
\text { Global }\end{array}$ & $\begin{array}{l}\text { Estudio } \\
\text { cuantitativo }\end{array}$ & $\begin{array}{l}\text { Describir el grado de sobrecarga y } \\
\text { deterioro de la salud mental en el } \\
\text { cuidador informal }\end{array}$ \\
\hline $\begin{array}{l}\text { Cano, B. G., García, I. } \\
\text { A., \& Bernal, E. R. M. } \\
\text { (2015) }\end{array}$ & $\begin{array}{l}\text { El sistema informal de } \\
\text { cuidados desde la } \\
\text { perspectiva de género: } \\
\text { Revisión bibliográfica }\end{array}$ & $\begin{array}{l}\text { Hygia de } \\
\text { enfermería: } \\
\text { revista } \\
\text { científica del } \\
\text { colegio }\end{array}$ & $\begin{array}{l}\text { Artículo de } \\
\text { revisión } \\
\text { (cualitativo) }\end{array}$ & $\begin{array}{l}\text { Conocer los cambios } \\
\text { sociodemográficos ocurridos en } \\
\text { España que generan nuevas } \\
\text { demandas de salud. Conocer el } \\
\text { sistema informal de cuidados y las } \\
\text { desigualdades desde la perspectiva } \\
\text { de género antes las nuevas } \\
\text { demandas de salud. Conocer el } \\
\text { perfil de las personas que prestan } \\
\text { cuidados informales }\end{array}$ \\
\hline $\begin{array}{l}\text { Martínez-Marcos, M., } \\
\text { \& la Cuesta-Benjumea, } \\
\text { D. (2015) }\end{array}$ & $\begin{array}{l}\text { Women's self- } \\
\text { management of chronic } \\
\text { illnesses in the context of } \\
\text { caregiving: A grounded } \\
\text { theory study }\end{array}$ & $\begin{array}{l}\text { Journal of } \\
\text { Clinical Nursing }\end{array}$ & $\begin{array}{l}\text { Estudio } \\
\text { cualitativo }\end{array}$ & $\begin{array}{l}\text { Descubrir como las mujeres } \\
\text { cuidadoras informales autogestionan } \\
\text { su propia enfermedad crónica }\end{array}$ \\
\hline $\begin{array}{l}\text { Ferré-Grau, C., Sevilla- } \\
\text { Casado, M., Lleixá- } \\
\text { Fortuño, M., Aparicio- } \\
\text { Casals, M. R., Cid- } \\
\text { Buera, D., Rodero- } \\
\text { Sánchez, V., \& Vives- } \\
\text { Relats, C. (2014) }\end{array}$ & $\begin{array}{l}\text { Effectiveness of problema } \\
\text { solving technique in caring } \\
\text { for family caregivers: a } \\
\text { clinical trial study in an } \\
\text { urban area of Catalonia } \\
\text { (Spain) }\end{array}$ & $\begin{array}{l}\text { Journal of } \\
\text { clinical nursing }\end{array}$ & Estudio mixto & $\begin{array}{l}\text { Evaluar la efectividad de la técnica } \\
\text { de resolución de problemas en la } \\
\text { reducción de los síntomas de } \\
\text { ansiedad y depresión entre los } \\
\text { cuidadores informales, describir y } \\
\text { evaluar el proceso llevado a cabo } \\
\text { por las enfermeras para encontrar }\end{array}$ \\
\hline
\end{tabular}




\begin{tabular}{|c|c|c|c|c|}
\hline & & & & puntos fuertes y áreas de mejora \\
\hline $\begin{array}{l}\text { Ferré-Grau, C., } \\
\text { Casado, M. S., Cid- } \\
\text { Buera, D., LLeixà- } \\
\text { Fortuño, M., Monteso- } \\
\text { Curto, P., \& } \\
\text { Berenguer-Poblet, M. } \\
\text { (2014) }\end{array}$ & $\begin{array}{l}\text { Caring for family } \\
\text { caregivers: An analysis of } \\
\text { a family-centered } \\
\text { intervention }\end{array}$ & $\begin{array}{l}\text { Revista da } \\
\text { Escola de } \\
\text { Enfermagem } \\
\text { da USP }\end{array}$ & Estudio mixto & $\begin{array}{l}\text { Evaluar la efectividad de la Técnica } \\
\text { de Resolución de Problemas (TRP) } \\
\text { en cuidadoras informales mediante } \\
\text { escalas positivas de ansiedad, } \\
\text { depresión y malestar emocional, } \\
\text { explorar los factores facilitadores y } \\
\text { barreras en su aplicación a partir de } \\
\text { la narrativa de las enfermeras }\end{array}$ \\
\hline $\begin{array}{l}\text { Del-Pino-Casado, R., } \\
\text { Millán-Cobo, M. D., } \\
\text { Palomino-Moral, P. A., } \\
\text { \& Frías-Osuna, A. } \\
(2014)\end{array}$ & $\begin{array}{l}\text { Cultural correlates of } \\
\text { burden in primary } \\
\text { caregivers of older } \\
\text { relatives: A cross sectional } \\
\text { study }\end{array}$ & $\begin{array}{l}\text { Journal of } \\
\text { Nursing } \\
\text { Scholarship }\end{array}$ & $\begin{array}{l}\text { Estudio } \\
\text { cuantitativo }\end{array}$ & $\begin{array}{l}\text { Analizar el efecto de los factores } \\
\text { culturales en la carga subjetiva de } \\
\text { cuidadores informales }\end{array}$ \\
\hline $\begin{array}{l}\text { Mendoza Sánchez, R. } \\
\text { M., Hernández García, } \\
\text { E. L., Medina Pérez, } \\
\text { M., Gómez Perera, M., } \\
\text { Estrada Suárez Pérez, } \\
\text { L., Navarro Vázquez, } \\
\text { F. J., \& Pérez, F. } \\
\text { (2014) }\end{array}$ & $\begin{array}{l}\text { Perfil del cuidador } \\
\text { principal en el área de } \\
\text { salud de Gran Canaria }\end{array}$ & $\begin{array}{l}\text { Ene: Revista } \\
\text { de Enfermería }\end{array}$ & $\begin{array}{l}\text { Estudio } \\
\text { cuantitativo }\end{array}$ & $\begin{array}{l}\text { Analizar el perfil de la persona } \\
\text { cuidadora informal }\end{array}$ \\
\hline $\begin{array}{l}\text { Rico-Blázquez, M., } \\
\text { Gómez, S. S., \& } \\
\text { Gallego, C. F. (2014) }\end{array}$ & $\begin{array}{l}\text { El cuidado como elemento } \\
\text { transversal en la atención } \\
\text { a pacientes crónicos } \\
\text { complejos }\end{array}$ & $\begin{array}{l}\text { Enfermería } \\
\text { Clínica }\end{array}$ & $\begin{array}{l}\text { Articulo de } \\
\text { revisión } \\
\text { (cualitativo) }\end{array}$ & $\begin{array}{l}\text { Analizar la situación de la atención } \\
\text { del paciente crónico }\end{array}$ \\
\hline $\begin{array}{l}\text { Rico-Blázquez, M., } \\
\text { Escortell-Mayor, E., } \\
\text { del-Cura-González, I., } \\
\text { Sanz-Cuesta, T., } \\
\text { Gallego-Berciano, P., } \\
\text { de las Casas-Cámara, } \\
\text { G.,... \& Domínguez- } \\
\text { Pérez, L. (2014) }\end{array}$ & $\begin{array}{l}\text { CuidaCare: effectiveness } \\
\text { of a nursing intervention } \\
\text { on the quality of life's } \\
\text { caregiver: cluster- } \\
\text { randomized clinical trial }\end{array}$ & BMC Nursing & $\begin{array}{l}\text { Estudio } \\
\text { cuantitativo }\end{array}$ & $\begin{array}{l}\text { Evaluar la eficacia de una } \\
\text { intervención de cuidados } \\
\text { en la atención primaria para mejorar } \\
\text { la calidad de vida de los cuidadores } \\
\text { informales }\end{array}$ \\
\hline $\begin{array}{l}\text { Rodríguez Ruiz, J. C., } \\
\text { Archilla Castillo, M. I., } \\
\text { \& Archilla Castillo, M. } \\
\text { (2014) }\end{array}$ & $\begin{array}{l}\text { La sobrecarga de los } \\
\text { cuidadores informales }\end{array}$ & $\begin{array}{l}\text { Metas de } \\
\text { Enfermería }\end{array}$ & $\begin{array}{l}\text { Estudio } \\
\text { cuantitativo }\end{array}$ & $\begin{array}{l}\text { Analizar la presencia de sobrecarga } \\
\text { en los cuidadores informales e } \\
\text { identificar algunas variables } \\
\text { asociadas que puedan influir en ella }\end{array}$ \\
\hline $\begin{array}{l}\text { Casado-Mejía, R., \& } \\
\text { Ruiz-Arias, E. (2013) }\end{array}$ & $\begin{array}{l}\text { Estrategias de provisión } \\
\text { de cuidados familiares a } \\
\text { personas mayores } \\
\text { dependientes }\end{array}$ & $\begin{array}{l}\text { Índex de } \\
\text { Enfermería }\end{array}$ & $\begin{array}{l}\text { Estudio } \\
\text { cualitativo }\end{array}$ & $\begin{array}{l}\text { Explorar, identificar y caracterizar las } \\
\text { distintas estrategias de provisión de } \\
\text { cuidados a personas dependientes } \\
\text { que desarrollan las personas } \\
\text { cuidadoras informales }\end{array}$ \\
\hline $\begin{array}{l}\text { Cuenca, R. M. A. } \\
\text { (2013) }\end{array}$ & $\begin{array}{l}\text { Historia de una Gran } \\
\text { Cuidadora: Cómo } \\
\text { gestionar mi vida }\end{array}$ & $\begin{array}{l}\text { Archivos de la } \\
\text { Memoria }\end{array}$ & $\begin{array}{l}\text { Relato } \\
\text { biográfico } \\
\text { (cualitativo) }\end{array}$ & $\begin{array}{l}\text { Conocer la vida y experiencia de una } \\
\text { cuidadora informal }\end{array}$ \\
\hline $\begin{array}{l}\text { Fernández Lao, I., } \\
\text { Silvano Arranz, A., \& } \\
\text { Pino Berenguer, M. D. } \\
\text { (2013) }\end{array}$ & $\begin{array}{l}\text { Percepción del cuidado } \\
\text { por parte del cuidador } \\
\text { familiar }\end{array}$ & $\begin{array}{l}\text { Índex de } \\
\text { Enfermería }\end{array}$ & $\begin{array}{l}\text { Estudio } \\
\text { cualitativo }\end{array}$ & $\begin{array}{l}\text { Determinar el significado del cuidado } \\
\text { en el cuidador informal }\end{array}$ \\
\hline $\begin{array}{l}\text { Gallart, A., Cruz, F., \& } \\
\text { Zabalegui, A. (2013). }\end{array}$ & $\begin{array}{l}\text { Factors influencing burden } \\
\text { among non professional } \\
\text { immigrant caregivers: a } \\
\text { case-control study }\end{array}$ & $\begin{array}{l}\text { Journal of } \\
\text { advanced } \\
\text { nursing }\end{array}$ & $\begin{array}{l}\text { Estudio } \\
\text { cuantitativo }\end{array}$ & $\begin{array}{l}\text { Identificar los factores relacionados } \\
\text { con la carga que es experimentada } \\
\text { por las cuidadoras informales } \\
\text { inmigrantes sin formación }\end{array}$ \\
\hline $\begin{array}{l}\text { Gallo Estrada, J., } \\
\text { Molina Mula, J., } \\
\text { Novajra, A. M., \& } \\
\text { Taltavull Aparicio, J. M. } \\
\text { (2013) }\end{array}$ & $\begin{array}{l}\text { Estrategias de cuidados } \\
\text { de las familias con las } \\
\text { personas mayores que } \\
\text { viven solas }\end{array}$ & $\begin{array}{l}\text { Índex de } \\
\text { Enfermería }\end{array}$ & $\begin{array}{l}\text { Estudio } \\
\text { cualitativo }\end{array}$ & $\begin{array}{l}\text { Conocer la percepción de la familia } \\
\text { sobre el cuidado a los mayores } \\
\text { dependientes }\end{array}$ \\
\hline García, D. V. (2013) & $\begin{array}{l}\text { Cuidador familiar y } \\
\text { profesional de enfermería: } \\
\text { dos perspectivas distintas } \\
\text { del cuidado }\end{array}$ & $\begin{array}{l}\text { Archivos de la } \\
\text { Memoria }\end{array}$ & $\begin{array}{l}\text { Relato } \\
\text { biográfico } \\
\text { (cualitativo) }\end{array}$ & $\begin{array}{l}\text { Describir la experiencia, dificultades, } \\
\text { sentimientos y aprendizajes de la } \\
\text { persona cuidadora informal y cómo } \\
\text { influye en su vida profesional }\end{array}$ \\
\hline $\begin{array}{l}\text { Guerra Martín, M. D., } \\
\text { \& Zambrano } \\
\text { Domínguez, E. M. } \\
\text { (2013) }\end{array}$ & $\begin{array}{l}\text { Relación entre los } \\
\text { problemas de salud de los } \\
\text { mayores dependientes y } \\
\text { la formación de los } \\
\text { cuidadores informales }\end{array}$ & $\begin{array}{l}\text { Enfermería } \\
\text { Global }\end{array}$ & $\begin{array}{l}\text { Estudio } \\
\text { cuantitativo }\end{array}$ & $\begin{array}{l}\text { Estudiar los problemas de salud de } \\
\text { los mayores dependientes y la } \\
\text { formación de los cuidadores } \\
\text { informales }\end{array}$ \\
\hline Mata, R. M. A. (2013) & $\begin{array}{l}\text { El cuidado de un familiar } \\
\text { anciano dependiente con } \\
\text { pluripatología }\end{array}$ & $\begin{array}{l}\text { Archivos de la } \\
\text { Memoria }\end{array}$ & $\begin{array}{l}\text { Estudio } \\
\text { cualitativo }\end{array}$ & $\begin{array}{l}\text { Conocer el impacto producido en la } \\
\text { vida de un cuidador informal y } \\
\text { comprender las diferencias que } \\
\text { existen con respecto al cuidado de } \\
\text { personas ajenas a la propia familia }\end{array}$ \\
\hline $\begin{array}{l}\text { Matute, A. E. G., } \\
\text { Barraso, M. D. C. P., } \\
\text { Osuna, M. E., \& } \\
\text { Jiménez, M. R. (2013) }\end{array}$ & $\begin{array}{l}\text { El manual de } \\
\text { recomendaciones como } \\
\text { herramienta de apoyo a la } \\
\text { persona cuidadora }\end{array}$ & $\begin{array}{l}\text { Evidentia: } \\
\text { Revista de } \\
\text { enfermería } \\
\text { basada en la }\end{array}$ & $\begin{array}{l}\text { Estudio } \\
\text { cualitativo }\end{array}$ & $\begin{array}{l}\text { Mostrar la experiencia en la } \\
\text { elaboración de un manual que } \\
\text { recoge las recomendaciones acerca } \\
\text { de los cuidados desarrollado por }\end{array}$ \\
\hline
\end{tabular}




\begin{tabular}{|c|c|c|c|c|}
\hline & & evidencia & & $\begin{array}{l}\text { cuidadoras informales en su } \\
\text { domicilio }\end{array}$ \\
\hline $\begin{array}{l}\text { Mejía, R. C., Arias, E. } \\
\text { R., Gallego, J. C., } \\
\text { Marín, M. J. A., \& } \\
\text { Sánchez, M. O. (2013) }\end{array}$ & $\begin{array}{l}\text { Validación y pilotaje de un } \\
\text { cuestionario con } \\
\text { perspectiva de género } \\
\text { para profundizar en el } \\
\text { cuidado familiar }\end{array}$ & $\begin{array}{l}\text { Evidentia: } \\
\text { Revista de } \\
\text { enfermería } \\
\text { basada en la } \\
\text { evidencia }\end{array}$ & $\begin{array}{l}\text { Articulo de } \\
\text { revisión } \\
\text { (cuantitativo) }\end{array}$ & $\begin{array}{l}\text { Diseñar y validar un cuestionario } \\
\text { para conocer el perfil de } \\
\text { cuidadoras/es informales, sus } \\
\text { estrategias de provisión de cuidados, } \\
\text { medidas institucionales elegidas y } \\
\text { repercusiones en salud }\end{array}$ \\
\hline $\begin{array}{l}\text { Muñoz, M. I. G., } \\
\text { Galiano, M. D. C. L., \& } \\
\text { Muñoz, I. M. G. (2013) }\end{array}$ & $\begin{array}{l}\text { Cuidar, un hábito familiar. } \\
\text { La satisfacción de cuidar a } \\
\text { los seres queridos }\end{array}$ & $\begin{array}{l}\text { Archivos de la } \\
\text { Memoria }\end{array}$ & $\begin{array}{l}\text { Relato } \\
\text { biográfico } \\
\text { (cualitativo) }\end{array}$ & $\begin{array}{l}\text { Describir la experiencia como } \\
\text { cuidadora informal }\end{array}$ \\
\hline $\begin{array}{l}\text { Sotto Mayor, M., } \\
\text { Sequeira, C., \& García, } \\
\text { B. (2013) }\end{array}$ & $\begin{array}{l}\text { Consulta de enfermería } \\
\text { dirigida a cuidadores } \\
\text { informales: instrumentos } \\
\text { de diagnóstico e } \\
\text { intervención }\end{array}$ & Gerokomos & $\begin{array}{l}\text { Estudio } \\
\text { cuantitativo }\end{array}$ & $\begin{array}{l}\text { Identificar las principales } \\
\text { necesidades de los cuidadores } \\
\text { informales que recurren a la consulta } \\
\text { de enfermería, las prácticas } \\
\text { implementadas y los resultados } \\
\text { obtenidos }\end{array}$ \\
\hline $\begin{array}{l}\text { Trigueros, A. C., } \\
\text { Montes, C. L. L., \& } \\
\text { Gallego, F. R. (2013) }\end{array}$ & $\begin{array}{l}\text { Nacida para cuidar. Relato } \\
\text { biográfico de una } \\
\text { adolescente }\end{array}$ & $\begin{array}{l}\text { Archivos de la } \\
\text { Memoria }\end{array}$ & $\begin{array}{l}\text { Relato } \\
\text { biográfico } \\
\text { (cualitativo) }\end{array}$ & $\begin{array}{l}\text { Explorar las vivencias, actitudes y } \\
\text { sentimientos que desarrolla un } \\
\text { adolescente al asumir su rol de } \\
\text { cuidadora familiar principal }\end{array}$ \\
\hline $\begin{array}{l}\text { Álvarez-Tello, M., } \\
\text { Casado-Mejía, R., } \\
\text { Ortega-Calvo, M., \& } \\
\text { Ruiz-Arias, E. (2012) }\end{array}$ & $\begin{array}{l}\text { Sobrecarga sentida en } \\
\text { personas cuidadoras } \\
\text { informales de pacientes } \\
\text { pluripatológicos en una } \\
\text { zona urbana }\end{array}$ & $\begin{array}{l}\text { Enfermería } \\
\text { Clínica }\end{array}$ & $\begin{array}{l}\text { Estudio } \\
\text { cuantitativo }\end{array}$ & $\begin{array}{l}\text { Determinar el perfil de la persona } \\
\text { cuidadora informal, ientificar factores } \\
\text { asociados a la sobrecarga sentida y } \\
\text { manifestaciones de cansancio para } \\
\text { construir } \\
\text { modelos predictivos mediante los } \\
\text { ítems del Îndice de Esfuerzo del } \\
\text { Cuidador }\end{array}$ \\
\hline $\begin{array}{l}\text { De la Cuesta- } \\
\text { Benjumea, C., Donet- } \\
\text { Montagut, T., \& de } \\
\text { Cádiz, M. J. G. G. } \\
\text { (2012) }\end{array}$ & $\begin{array}{l}\text { Turning to One's Own } \\
\text { World" Escape } \\
\text { Mechanisms Employed by } \\
\text { Immigrant Caregivers in } \\
\text { Spain for Relieving the } \\
\text { Burden of Care }\end{array}$ & $\begin{array}{l}\text { Journal of } \\
\text { Transcultural } \\
\text { Nursing }\end{array}$ & $\begin{array}{l}\text { Estudio } \\
\text { cualitativo }\end{array}$ & $\begin{array}{l}\text { Analizar las estrategias que utilizan } \\
\text { los cuidadores informales de origen } \\
\text { inmigrante para aliviar la carga del } \\
\text { cuidado }\end{array}$ \\
\hline $\begin{array}{l}\text { Del-Pino Casado, R., } \\
\text { Frías-Osuna, A., } \\
\text { Palomino-Moral, P. A., } \\
\text { \& Ramón Martínez- } \\
\text { Riera, J. (2012) }\end{array}$ & $\begin{array}{l}\text { Gender differences } \\
\text { regarding informal } \\
\text { caregivers of older people }\end{array}$ & $\begin{array}{l}\text { Journal of } \\
\text { Nursing } \\
\text { Scholarship }\end{array}$ & $\begin{array}{l}\text { Estudio } \\
\text { cuantitativo }\end{array}$ & $\begin{array}{l}\text { Examinar las diferencias } \\
\text { relacionadas con el género entre los } \\
\text { cuidadores informales de personas } \\
\text { en situación de dependencia }\end{array}$ \\
\hline Iglesias, L. R. (2012) & $\begin{array}{l}\text { El cuidado familiar: } \\
\text { Percepciones, } \\
\text { repercusiones y } \\
\text { emociones de una } \\
\text { persona cuidadora }\end{array}$ & $\begin{array}{l}\text { Archivos de la } \\
\text { Memoria }\end{array}$ & $\begin{array}{l}\text { Relato } \\
\text { biográfico } \\
\text { (cualitativo) }\end{array}$ & $\begin{array}{l}\text { Reconocer la tarea de las personas } \\
\text { cuidadoras informales }\end{array}$ \\
\hline $\begin{array}{l}\text { Martín, M. D. S. M., } \\
\text { Rodríguez, A. A., \& } \\
\text { Serrano, R. R. (2012) }\end{array}$ & $\begin{array}{l}\text { Una vida dedicada al } \\
\text { cuidado. El relato de una } \\
\text { cuidadora profesional y } \\
\text { familiar }\end{array}$ & $\begin{array}{l}\text { Archivos de la } \\
\text { Memoria }\end{array}$ & $\begin{array}{l}\text { Relato } \\
\text { biográfico } \\
\text { (cualitativo) }\end{array}$ & $\begin{array}{l}\text { Presentar la perspectiva de género } \\
\text { en el cuidado informal desde la } \\
\text { mirada de una enfermera }\end{array}$ \\
\hline $\begin{array}{l}\text { Vázquez-Sánchez, M. } \\
\text { Á., Aguilar-Trujillo, M. } \\
\text { P., Estébanez- } \\
\text { Carvajal, F. M., } \\
\text { Casals-Vázquez, C., } \\
\text { Casals-Sánchez, J. L., } \\
\text { \& Heras-Pérez, M. C. } \\
\text { (2012) }\end{array}$ & $\begin{array}{l}\text { Influencia de los } \\
\text { pensamientos } \\
\text { disfuncionales en la } \\
\text { sobrecarga de los } \\
\text { cuidadores de personas } \\
\text { dependientes }\end{array}$ & $\begin{array}{l}\text { Enfermería } \\
\text { Clínica }\end{array}$ & $\begin{array}{l}\text { Estudio } \\
\text { cuantitativo }\end{array}$ & $\begin{array}{l}\text { Conocer el nivel de pensamientos } \\
\text { disfuncionales en los cuidadores } \\
\text { informales y la relación que tienen } \\
\text { con la sobrecarga en el desempeño } \\
\text { del rol de cuidador }\end{array}$ \\
\hline $\begin{array}{l}\text { Villarejo Aguilar, L., } \\
\text { Peña, Z., \& Casado } \\
\text { Ponce, G. (2012) }\end{array}$ & $\begin{array}{l}\text { Sobrecarga y dolor } \\
\text { percibido en cuidadoras } \\
\text { de ancianos dependientes }\end{array}$ & $\begin{array}{l}\text { Enfermería } \\
\text { Global }\end{array}$ & $\begin{array}{l}\text { Estudio } \\
\text { cuantitativo }\end{array}$ & $\begin{array}{l}\text { Evaluar la sobrecarga subjetiva y el } \\
\text { dolor percibido en cuidadoras } \\
\text { informales de personas } \\
\text { dependientes y determinar si la } \\
\text { intensidad del dolor percibido está } \\
\text { asociado a la sobrecarga de la } \\
\text { cuidadora }\end{array}$ \\
\hline $\begin{array}{l}\text { Zambrano-Domínguez, } \\
\text { E. M., \& Guerra- } \\
\text { Martín, M. D. (2012) }\end{array}$ & $\begin{array}{l}\text { Formación del cuidador } \\
\text { informal: relación con el } \\
\text { tiempo de cuidado a } \\
\text { personas dependientes } \\
\text { mayores de } 65 \text { años }\end{array}$ & Aquichán & $\begin{array}{l}\text { Estudio } \\
\text { cuantitativo }\end{array}$ & $\begin{array}{l}\text { Analizar la formación recibida de } \\
\text { los/as cuidadores/as informales de } \\
\text { personas dependientes en relación } \\
\text { con el tiempo de cuidado }\end{array}$ \\
\hline Bover, A. (2011) & $\begin{array}{l}\text { Economic crisis, austerity } \\
\text { discourses and caregiving: } \\
\text { how to remain relevant } \\
\text { through engagement and } \\
\text { social justice }\end{array}$ & Nursing Inquiry & $\begin{array}{l}\text { Artículo de } \\
\text { reflexión }\end{array}$ & $\begin{array}{l}\text { Estudiar como las medidas aplicadas } \\
\text { para el control del gasto en salud } \\
\text { pública han afectado la salud y la } \\
\text { equidad dentro del sistema público } \\
\text { de salud }\end{array}$ \\
\hline $\begin{array}{l}\text { Del-Pino-Casado, R., } \\
\text { Frías-Osuna, A., } \\
\text { Palomino Moral, PA, y }\end{array}$ & $\begin{array}{l}\text { Coping and subjective } \\
\text { burden in caregivers of } \\
\text { older relatives: a }\end{array}$ & $\begin{array}{l}\text { Journal of } \\
\text { Advanced } \\
\text { Nursing }\end{array}$ & $\begin{array}{l}\text { Artículo de } \\
\text { revisión } \\
\text { (cuantitativo) }\end{array}$ & $\begin{array}{l}\text { Analizar el efecto de las estrategias } \\
\text { de afrontamiento sobre la carga } \\
\text { subjetiva de los cuidadores }\end{array}$ \\
\hline
\end{tabular}




\begin{tabular}{|c|c|c|c|c|}
\hline $\begin{array}{l}\text { Pancorbo-Hidalgo, PL } \\
\text { (2011) }\end{array}$ & $\begin{array}{l}\text { quantitative systemative } \\
\text { review }\end{array}$ & & & informales \\
\hline $\begin{array}{l}\text { del-Pino-Casado, R., } \\
\text { Frías-Osuna, A., \& } \\
\text { Palomino-Moral, P. A. } \\
\text { (2011) }\end{array}$ & $\begin{array}{l}\text { Subjective burden and } \\
\text { cultural motives for } \\
\text { caregiving in informal } \\
\text { caregivers of older people }\end{array}$ & $\begin{array}{l}\text { Journal of } \\
\text { Nursing } \\
\text { Scholarship }\end{array}$ & $\begin{array}{l}\text { Estudio } \\
\text { cuantitativo }\end{array}$ & $\begin{array}{l}\text { Investigar las variables relacionadas } \\
\text { con los motivos del cuidado } \\
\text { informal/cultural y analizar su } \\
\text { relación con la carga subjetiva de los } \\
\text { cuidadores informales }\end{array}$ \\
\hline $\begin{array}{l}\text { Germán Bes, C., } \\
\text { Hueso Navarro, F., \& } \\
\text { Huércanos Esparza, I. } \\
\text { (2011) }\end{array}$ & $\begin{array}{l}\text { El cuidado en peligro en la } \\
\text { sociedad global }\end{array}$ & $\begin{array}{l}\text { Enfermería } \\
\text { Global }\end{array}$ & $\begin{array}{l}\text { Artículo de } \\
\text { reflexión }\end{array}$ & $\begin{array}{l}\text { Investigar sobre el riesgo de la } \\
\text { pérdida de los cuidados informales, } \\
\text { invisibles o humanizados, tanto en la } \\
\text { sociedad como en la profesión } \\
\text { enfermera }\end{array}$ \\
\hline $\begin{array}{l}\text { Vives-Relats, C., } \\
\text { Ferré-Grau, C., } \\
\text { Rodero-Sánchez, V., } \\
\text { Cid-Buera, D., \& } \\
\text { Miembros del Grupo } \\
\text { de Investigación: } \\
\text { Enfermería Aplicada } \\
\text { de la URV. (2011) }\end{array}$ & $\begin{array}{l}\text { Cuidar a una cuidadora } \\
\text { familiar en Atención } \\
\text { Primaria a partir de la } \\
\text { teoría de la incertidumbre }\end{array}$ & $\begin{array}{l}\text { Enfermería } \\
\text { Clínica }\end{array}$ & $\begin{array}{l}\text { Estudio } \\
\text { cualitativo }\end{array}$ & $\begin{array}{l}\text { Abordar los fenómenos que viven las } \\
\text { cuidadoras informales desde las } \\
\text { perspectivas de sus vivencias y } \\
\text { estrés }\end{array}$ \\
\hline Crespo, E. M. (2010) & $\begin{array}{l}\text { Valoración del apoyo } \\
\text { socio-familiar de las } \\
\text { cuidadoras en una zona } \\
\text { básica de salud }\end{array}$ & $\begin{array}{l}\text { Evidentia: } \\
\text { Revista de } \\
\text { enfermería } \\
\text { basada en la } \\
\text { evidencia }\end{array}$ & $\begin{array}{l}\text { Estudio } \\
\text { cuantitativo }\end{array}$ & $\begin{array}{l}\text { Valorar el apoyo social y familiar de } \\
\text { las cuidadoras informales de } \\
\text { personas dependientes }\end{array}$ \\
\hline $\begin{array}{l}\text { Delicado Useros, M. } \\
\text { V., Alcarria Rozalén, } \\
\text { A., Ortega Martínez, } \\
\text { C., Alfaro Espín, A., } \\
\text { García Alcaraz, F., \& } \\
\text { Candel Parra, E. } \\
\text { (2010) }\end{array}$ & $\begin{array}{l}\text { Autoestima, apoyo familiar } \\
\text { y social en cuidadores } \\
\text { familiares de personas } \\
\text { dependientes }\end{array}$ & $\begin{array}{l}\text { Metas de } \\
\text { Enfermería }\end{array}$ & $\begin{array}{l}\text { Estudio } \\
\text { cuantitativo }\end{array}$ & $\begin{array}{l}\text { Describir perfil de cuidadoras } \\
\text { informales, características del } \\
\text { cuidado, identificar apoyo recibido }\end{array}$ \\
\hline $\begin{array}{l}\text { Rogero-García, J. } \\
(2010)\end{array}$ & $\begin{array}{l}\text { Las consecuencias del } \\
\text { cuidado familiar sobre el } \\
\text { cuidador: Una valoración } \\
\text { compleja y necesaria }\end{array}$ & $\begin{array}{l}\text { Índex de } \\
\text { Enfermería }\end{array}$ & $\begin{array}{l}\text { Artículo de } \\
\text { reflexión }\end{array}$ & $\begin{array}{l}\text { Elaborar una propuesta de } \\
\text { clasificación de las consecuencias } \\
\text { del cuidar sobre el cuidador informal }\end{array}$ \\
\hline $\begin{array}{l}\text { Rogero-García, J., \& } \\
\text { Martín-Coppola, E. } \\
(2010)\end{array}$ & $\begin{array}{l}\text { Un estudio exploratorio } \\
\text { del cuidado provisto por } \\
\text { inmigrantes a personas } \\
\text { mayores de } 64 \text { años en } \\
\text { los hogares }\end{array}$ & $\begin{array}{l}\text { Índex de } \\
\text { Enfermería }\end{array}$ & $\begin{array}{l}\text { Estudio } \\
\text { cuantitativo }\end{array}$ & $\begin{array}{l}\text { Explorar el tipo de atención que } \\
\text { proveen los cuidadores informales } \\
\text { inmigrantes a la persona en } \\
\text { situación de dependencia en los } \\
\text { hogares }\end{array}$ \\
\hline $\begin{array}{l}\text { Rodríguez, P. H., } \\
\text { Sampedro, M. A. Z., \& } \\
\text { Zamudio, E. F. (2010) }\end{array}$ & $\begin{array}{l}\text { Sentimientos y emociones } \\
\text { en cuidadores informales }\end{array}$ & $\begin{array}{l}\text { Hygia de } \\
\text { enfermería: } \\
\text { revista } \\
\text { científica del } \\
\text { colegio }\end{array}$ & $\begin{array}{l}\text { Estudio } \\
\text { cualitativo }\end{array}$ & $\begin{array}{l}\text { Comprender las vivencias } \\
\text { analizando cómo la persona percibe } \\
\text { y comprende sus experiencias como } \\
\text { cuidadora informal }\end{array}$ \\
\hline $\begin{array}{l}\text { Alvaro, A. M. B., } \\
\text { López, M. C. O., } \\
\text { Bustillo, M. G., } \\
\text { Fernández, M. Á. G., } \\
\text { Gómez, M. B. S., \& } \\
\text { Climents, G. D. (2009) }\end{array}$ & $\begin{array}{l}\text { Educación grupal versus } \\
\text { educación individual en } \\
\text { cuidadores familiares. } \\
\text { Revisión Sistemática }\end{array}$ & $\begin{array}{l}\text { Evidentia: } \\
\text { Revista de } \\
\text { enfermería } \\
\text { basada en la } \\
\text { evidencia }\end{array}$ & $\begin{array}{l}\text { Artículo de } \\
\text { revisión } \\
\text { (cuantitativo) }\end{array}$ & $\begin{array}{l}\text { Encontrar evidencias que permitan } \\
\text { identificar el instrumento de medida } \\
\text { de la carga del cuidador informal }\end{array}$ \\
\hline $\begin{array}{l}\text { Delgado González, E., } \\
\text { González Esteban, M. } \\
\text { P., Ballesteros Alvaro, } \\
\text { A. M., Pérez-Alonso, } \\
\text { J., Mediavilla, M. E., } \\
\text { Aragón Posadas, R., ... } \\
\text { \& Guzmán-Fernández, } \\
\text { M. A. (2009) }\end{array}$ & $\begin{array}{l}\text { ¿Existen instrumentos } \\
\text { válidos para medir el } \\
\text { síndrome del cuidador } \\
\text { familiar? Una revisión } \\
\text { sistemática de la literatura }\end{array}$ & $\begin{array}{l}\text { Banco de } \\
\text { evidencias de } \\
\text { Sacyl }\end{array}$ & $\begin{array}{l}\text { Artículo de } \\
\text { revisión } \\
\text { (cuantitativo) }\end{array}$ & $\begin{array}{l}\text { Identificar la mejor evidencia } \\
\text { disponible sobre EpS grupal versus } \\
\text { individual en cuidadores informales } \\
\text { de personas dependientes para } \\
\text { elaborar recomendaciones para } \\
\text { mejorar la atención }\end{array}$ \\
\hline $\begin{array}{l}\text { González-Pisano, A. } \\
\text { C., Granado-Villacé, } \\
\text { R., García-Jáñez, E., } \\
\text { del Cano-González, } \\
\text { C., \& Fernández- } \\
\text { Fernández, M. I. } \\
\text { (2009) }\end{array}$ & $\begin{array}{l}\text { Calidad de vida } \\
\text { relacionada con la salud } \\
\text { en cuidadoras de } \\
\text { personas dependientes de } \\
\text { dos zonas rurales de León }\end{array}$ & $\begin{array}{l}\text { Enfermería } \\
\text { Clínica }\end{array}$ & $\begin{array}{l}\text { Estudio } \\
\text { cuantitativo }\end{array}$ & $\begin{array}{l}\text { Estudiar la calidad de vida } \\
\text { relacionada con la salud y la relación } \\
\text { con el apoyo social, la función } \\
\text { familiar y el nivel de carga de } \\
\text { cuidadoras informales de personas } \\
\text { con dependencia }\end{array}$ \\
\hline $\begin{array}{l}\text { González-Valentín, A., } \\
\text { \& Gálvez-Romero, C. } \\
\text { (2009) }\end{array}$ & $\begin{array}{l}\text { Características } \\
\text { sociodemográficas, de } \\
\text { salud y utilización de } \\
\text { recursos sanitarios de } \\
\text { cuidadores de ancianos } \\
\text { atendidos en domicilio }\end{array}$ & Gerokomos & $\begin{array}{l}\text { Estudio } \\
\text { cuantitativo }\end{array}$ & $\begin{array}{l}\text { Describir las características } \\
\text { sociodemográficas y de salud de los } \\
\text { cuidadores informales para } \\
\text { establecer factores } \\
\text { determinantes del estado de salud y } \\
\text { utilización } \\
\text { de recursos sanitarios de estos } \\
\text { cuidadores }\end{array}$ \\
\hline $\begin{array}{l}\text { López-Casanova, P., } \\
\text { Rodríguez-Palma, M., }\end{array}$ & $\begin{array}{l}\text { Perfil social de los } \\
\text { cuidadores familiares de }\end{array}$ & Gerokomos & $\begin{array}{l}\text { Estudio } \\
\text { cuantitativo }\end{array}$ & $\begin{array}{l}\text { Describir el perfil social de } \\
\text { los cuidadores informales y conocer }\end{array}$ \\
\hline
\end{tabular}




\begin{tabular}{|c|c|c|c|c|}
\hline $\begin{array}{l}\text { \& Herrero-Díaz, M. A. } \\
(2009)\end{array}$ & $\begin{array}{l}\text { pacientes dependientes } \\
\text { ingresados en el Hospital } \\
\text { General Universitario de } \\
\text { Elche }\end{array}$ & & & el tipo de necesidades que cubren \\
\hline $\begin{array}{l}\text { Bonet, I. Ú., \& Roger, } \\
\text { M. R. (2008) }\end{array}$ & $\begin{array}{l}\text { ¿Cómo repercute el cuidar } \\
\text { en los cuidadores } \\
\text { familiares de personas } \\
\text { dependientes? }\end{array}$ & $\begin{array}{l}\text { Nursing (Ed. } \\
\text { española) }\end{array}$ & $\begin{array}{l}\text { Artículo de } \\
\text { reflexión }\end{array}$ & $\begin{array}{l}\text { Analizar el impacto del cuidar en } \\
\text { cuidadores informales de personas } \\
\text { en situación de dependencia }\end{array}$ \\
\hline $\begin{array}{l}\text { Cuesta Benjumea, C. } \\
\text { D. L. (2008) }\end{array}$ & $\begin{array}{l}\text { Aliviar el peso del cuidado } \\
\text { familiar: Una revisión de la } \\
\text { bibliografía }\end{array}$ & $\begin{array}{l}\text { Índex de } \\
\text { Enfermería }\end{array}$ & $\begin{array}{l}\text { Artículo de } \\
\text { revisión } \\
\text { (cualitativo) }\end{array}$ & $\begin{array}{l}\text { Analizar los programas de respiro } \\
\text { dirigidos a cuidadores informales }\end{array}$ \\
\hline $\begin{array}{l}\text { De Cádiz, M. J. G. G., } \\
\text { de la Cuesta- } \\
\text { Benjumea, C., \& } \\
\text { Donet-Montagut, T. } \\
\text { (2008) }\end{array}$ & $\begin{array}{l}\text { Cuidadoras inmigrantes: } \\
\text { características del cuidado } \\
\text { que prestan a la } \\
\text { dependencia }\end{array}$ & $\begin{array}{l}\text { Enfermería } \\
\text { Clínica }\end{array}$ & $\begin{array}{l}\text { Artículo de } \\
\text { revisión } \\
\text { (cualitativo) }\end{array}$ & $\begin{array}{l}\text { Reflejar las características del } \\
\text { cuidado llevado a cabo por mujeres } \\
\text { de origen inmigrante }\end{array}$ \\
\hline $\begin{array}{l}\text { Escuredo Rodríguez, } \\
\text { B. (2008) }\end{array}$ & $\begin{array}{l}\text { Políticas de dependencia. } \\
\text { Consecuencias para las } \\
\text { familias }\end{array}$ & $\begin{array}{l}\text { Revista Rol de } \\
\text { Enfermería }\end{array}$ & $\begin{array}{l}\text { Estudio } \\
\text { cualitativo }\end{array}$ & $\begin{array}{l}\text { Analizar las principales políticas de } \\
\text { atención a la dependencia }\end{array}$ \\
\hline $\begin{array}{l}\text { Leyva-Moral, J. M., \& } \\
\text { Mogeda-Marina, N. } \\
(2008)\end{array}$ & $\begin{array}{l}\text { Necesidades } \\
\text { psicosociales del cuidador } \\
\text { informal }\end{array}$ & $\begin{array}{l}\text { Revista Rol de } \\
\text { Enfermería }\end{array}$ & Estudio mixto & $\begin{array}{l}\text { Proponer la utilización de los } \\
\text { diagnósticos de enfermería en la } \\
\text { planificación de cuidados }\end{array}$ \\
\hline $\begin{array}{l}\text { Torres Egea, M., } \\
\text { Ballesteros Pérez, E., } \\
\text { \& Sánchez Castillo, P. } \\
\text { D. (2008) }\end{array}$ & $\begin{array}{l}\text { Programas e } \\
\text { intervenciones de apoyo a } \\
\text { los cuidadores informales } \\
\text { en España }\end{array}$ & Gerokomos & $\begin{array}{l}\text { Artículo de } \\
\text { revisión } \\
\text { (cuantitativo) }\end{array}$ & $\begin{array}{l}\text { Analizar las publicaciones científicas } \\
\text { que tratan sobre diferentes } \\
\text { programas e intervenciones de } \\
\text { soporte a los cuidadores informales }\end{array}$ \\
\hline $\begin{array}{l}\text { Zabalegui, A., Bover, } \\
\text { A., Rodriquez, E., } \\
\text { Cabrera, E., Diaz, M., } \\
\text { Gallart, A.,... \& Pulpón, } \\
\text { A. M. (2008) }\end{array}$ & $\begin{array}{l}\text { Informal caregiving: } \\
\text { perceived needs }\end{array}$ & $\begin{array}{l}\text { Nursing } \\
\text { Science } \\
\text { Quarterly }\end{array}$ & $\begin{array}{l}\text { Estudio } \\
\text { cualitativo }\end{array}$ & $\begin{array}{l}\text { Conocer el perfil y analizar las } \\
\text { necesidades de las personas } \\
\text { cuidadoras informales }\end{array}$ \\
\hline $\begin{array}{l}\text { Camero, M. L. R., } \\
\text { Camero, N. R., } \\
\text { Hernández, R. A., } \\
\text { López, A. T., Salvador, } \\
\text { M. D. M. R., \& Ronda, } \\
\text { F. J. M. (2007) }\end{array}$ & $\begin{array}{l}\text { Mujeres, salud y cuidados } \\
\text { familiares. Instituciones } \\
\text { económicas desde la } \\
\text { perspectiva antropológica }\end{array}$ & $\begin{array}{l}\text { Índex de } \\
\text { Enfermería }\end{array}$ & $\begin{array}{l}\text { Artículo de } \\
\text { reflexión }\end{array}$ & $\begin{array}{l}\text { Analizar los aspectos económicos } \\
\text { del cuidar y su relación con el } \\
\text { género }\end{array}$ \\
\hline $\begin{array}{l}\text { González, M. A. O., } \\
\text { Muñiz, R. M. R., \& } \\
\text { Tinoco, J. P. (2007) }\end{array}$ & $\begin{array}{l}\text { Atención al cuidador/a } \\
\text { principal desde Atención } \\
\text { Primaria de salud }\end{array}$ & $\begin{array}{l}\text { Metas de } \\
\text { Enfermería }\end{array}$ & Estudio mixto & $\begin{array}{l}\text { Dar respuesta a la problemática de } \\
\text { los cuidadores informales a través } \\
\text { de talleres de formación }\end{array}$ \\
\hline Rodilla, J. M. (2007) & $\begin{array}{l}\text { Cuidar a las personas de } \\
\text { apoyo }\end{array}$ & $\begin{array}{l}\text { Metas de } \\
\text { Enfermería }\end{array}$ & Estudio mixto & $\begin{array}{l}\text { Proponer de intervención enfermera } \\
\text { a las personas cuidadoras } \\
\text { informales }\end{array}$ \\
\hline $\begin{array}{l}\text { Zabalegui Yarnoz, A., } \\
\text { Juandó-Prats, C., } \\
\text { Sáenz de Ormijana } \\
\text { Hernández, A., } \\
\text { Ramírez Llaras, A. M., } \\
\text { Pulpón Segura, A., } \\
\text { López Rodríguez, L., \& } \\
\text { Valentín, G. (2007) }\end{array}$ & $\begin{array}{l}\text { Los cuidadores informales } \\
\text { en España: perfil y } \\
\text { cuidados prestados }\end{array}$ & $\begin{array}{l}\text { Revista Rol de } \\
\text { Enfermería }\end{array}$ & $\begin{array}{l}\text { Artículo de } \\
\text { revisión } \\
\text { (cuantitativo) }\end{array}$ & $\begin{array}{l}\text { Analizar las publicaciones sobre la } \\
\text { situación de los cuidadores } \\
\text { informales, el perfil y tipo de cuidado }\end{array}$ \\
\hline $\begin{array}{l}\text { Gutiérrez Valverde, P., } \\
\text { \& Nieto Jiménez, P. } \\
(2007)\end{array}$ & $\begin{array}{l}\text { Nuevas cuidadoras. } \\
\text { ¿Cómo formarlas? }\end{array}$ & $\begin{array}{l}\text { Revista Rol de } \\
\text { Enfermería }\end{array}$ & Estudio mixto & $\begin{array}{l}\text { Dar respuesta a las necesidades de } \\
\text { formación a cuidadoras informales a } \\
\text { través de talleres formativos }\end{array}$ \\
\hline
\end{tabular}

Fuente. Elaboración propia.

Se presentan a continuación los resultados del análisis cuantitativo y cualitativo, en aras de responder a las preguntas planteadas.

Los principales resultados del análisis cuantitativo, cuyo objetivo fue cuantificar la producción científica en función de la tipología (Tabla II). 
Tabla II: Tipología de los artículos publicados.

\begin{tabular}{|c|c|c|c|c|c|c|c|c|c|c|c|}
\hline $\begin{array}{l}\text { Tipo de } \\
\text { artículo }\end{array}$ & $\begin{array}{l}\text { Año } \\
2007\end{array}$ & $\begin{array}{l}\text { Año } \\
2008\end{array}$ & $\begin{array}{l}\text { Año } \\
2009\end{array}$ & $\begin{array}{l}\text { Año } \\
2010\end{array}$ & $\begin{array}{l}\text { Año } \\
2011\end{array}$ & $\begin{array}{l}\text { Año } \\
2012 \\
\end{array}$ & $\begin{array}{l}\text { Año } \\
2013\end{array}$ & $\begin{array}{l}\text { Año } \\
2014\end{array}$ & $\begin{array}{l}\text { Año } \\
2015\end{array}$ & $\begin{array}{l}\text { Año } \\
2016 \\
\end{array}$ & Total \\
\hline $\begin{array}{l}\text { Articulo original } \\
\text { cualitativo }\end{array}$ & & 2 & & 1 & 1 & 1 & 4 & & 1 & 1 & 11 \\
\hline $\begin{array}{l}\text { Articulo original } \\
\text { mixto }\end{array}$ & 3 & 1 & & & & & & 2 & & & 6 \\
\hline $\begin{array}{l}\text { Artículo revisión } \\
\text { cuantitativo }\end{array}$ & 1 & 1 & 2 & & 1 & & 1 & & & & 6 \\
\hline $\begin{array}{l}\text { Artículo de } \\
\text { reflexión }\end{array}$ & 1 & 1 & & 1 & 2 & & & & & & 5 \\
\hline $\begin{array}{l}\text { Relato } \\
\text { biográfico }\end{array}$ & & & & & & 2 & 4 & & & & 6 \\
\hline TOTAL & 5 & 7 & 5 & 5 & 5 & 8 & 13 & 7 & 2 & 2 & 59 \\
\hline
\end{tabular}

Fuente. Elaboración propia.

El número de publicaciones relacionadas con el tema de investigación se mantuvo estable durante el período de análisis, con un ligero aumento durante los años 2012 y 2013, disminuyendo de forma significativa en los años 2015 y 2016. Se observa que el tipo de artículo más publicado entre 2007 y 2016 fue el basado en un estudio original cuantitativo, con un total de 21 artículos editados, seguido del artículo original basado en un estudio cualitativo, con un total de 11. La tipología de artículo menos recurrente fue la de artículo de revisión cualitativa, con 4 artículos.

Los artículos basados en estudios cuantitativos aportan sobre todo datos descriptivos sobre el perfil de las personas cuidadoras, instrumentos y escalas de medida de la sobrecarga resultante del cuidado y valoraciones de intervenciones enfermeras. Que éste sea el tipo de artículo publicado con más frecuencia es coherente con el hecho de que históricamente la enfermería ha apostado por diseños experimentales, recogidas de datos a través de encuestas y cuestionarios estandarizados y técnicas estadísticas de análisis de datos.

En el transcurso del análisis se observó que en las investigaciones cualitativas de carácter descriptivo los relatos biográficos e historias de vida se encuentran entre las técnicas más utilizadas con la finalidad de recoger en primera persona las experiencias de las personas cuidadoras. Estos relatos ponen de manifiesto cuestiones tan diversas como el impacto del cuidado, la satisfacción personal de cubrir las necesidades de sus familiares, las cargas diferenciales de cuidado entre hombres y mujeres, así como la necesidad de medidas sociales y sanitarias que mejoren su calidad de vida. Otras técnicas cualitativas utilizadas son los grupos focales, los grupos de discusión y las entrevistas semiestructuradas y/o en profundidad. A partir de éstas se pone de manifiesto que existen múltiples estrategias para organizar los cuidados informales y que las personas cuidadoras no se sienten apoyadas por las políticas públicas. La investigación cualitativa, en este sentido, ofrece gran potencial a la profesión enfermera para identificar las necesidades 
subjetivas de cuidado, para ofrecer una atención más individualizada y para crear nuevas opciones de investigación.

El objetivo del análisis cualitativo realizado en el presente trabajo fue caracterizar la producción científica en función de sus temáticas, cabe destacar tres temas relevantes (Figura 1).

Figura 1: Temas principales de los artículos publicados.

\begin{tabular}{|c|c|c|}
\hline $\begin{array}{c}\text { Estudio del perfil de la } \\
\text { persona cuidadora y la } \\
\text { consecuencia del cuidado }\end{array}$ & $\begin{array}{c}\text { Propuestas promoción del } \\
\text { cuidado y el autocuidado y } \\
\text { la prevención de la } \\
\text { sobrecarga del cuidado }\end{array}$ & $\begin{array}{c}\text { Utilización de herramientas } \\
\text { de valoración para la } \\
\text { planificación de la atención } \\
\text { a la persona cuidadora }\end{array}$ \\
\hline $\begin{array}{c}\text { Estudio del perfil de la } \\
\text { persona cuidadora }\end{array}$ & $\begin{array}{c}\text { Formación para el cuidado a la } \\
\text { persona cuidadora }\end{array}$ & $\begin{array}{c}\text { Valoración el cuidado } \\
\text { aportado por la persona } \\
\text { cuidadora }\end{array}$ \\
\hline $\begin{array}{c}\text { Estudio de la consecuencia } \\
\text { del cuidado en la persona }\end{array}$ & $\begin{array}{c}\text { Formación para el autocuidado } \\
\text { a la persona cuidadora }\end{array}$ & $\begin{array}{c}\text { Valoración y planificación de } \\
\text { las intervenciones y la } \\
\text { atención para la persona } \\
\text { cuidadora }\end{array}$ \\
\hline $\begin{array}{c}\text { Estudio de la prevención de } \\
\text { la sobrecarga del cuidado } \\
\text { en la persona cuidadora }\end{array}$ & $\begin{array}{c}\text { Intervenciones para la } \\
\text { prevención de la sobrecarga } \\
\text { del cuidado en la persona } \\
\text { cuidadora }\end{array}$ & \\
\hline
\end{tabular}

Fuente. Elaboración propia.

El primer tema predominante en las publicaciones analizadas lo constituye el perfil de la persona cuidadora y las consecuencias del cuidado sobre ella, lo cual se considera relevante a la hora de proponer actividades preventivas. Al estudio del perfil de la persona cuidadora las publicaciones añaden el de los impactos del cuidado sobre ella, clasificados como positivos o negativos. Los positivos incluyen el desarrollo o crecimiento personal de la persona cuidadora y la oportunidad de tener empleo. Los negativos, más numerosos y frecuentes, engloban un importante estrés crónico que afecta a la salud física, psíquica y calidad de vida del cuidador, así como las cuestiones económicas, laborales y de relación social.

Un segundo tema detectado radica en la intervención para la promoción del cuidado y el autocuidado y la prevención de la sobrecarga resultante del cuidado. Se razona la importancia de tener en cuenta a la persona cuidadora e identificar estrategias destinadas a ésta, ya que ello puede contribuir a su control sobre su calidad de vida y su propia salud, así pues, la principal tarea de las enfermeras, con las personas cuidadoras, consiste en la formación y el asesoramiento que tienen como objetivo ampliar conocimientos y habilidades en el cuidado y el autocuidado.

El tercer y último tema hace referencia a la utilización de herramientas de valoración para la planificación de la atención a la persona cuidadora. Se propone el uso del lenguaje enfermero estandarizado basado en los criterios de la North American Nursing Association (NANDA). El uso de los diagnósticos de enfermería como herramienta de planificación de objetivos e intervenciones unifica y asegura un entendimiento entre profesionales, teniendo en cuenta la elaboración y uso de los manuales de recomendaciones en la práctica diaria con una metodología del cuidado que permita acercarse a una visión global de las necesidades de las personas y mejorar la calidad de la atención a éstas. 


\section{DISCUSIÓN}

Las publicaciones analizadas reflejan en gran medida el giro asistencial en el ámbito de la cronicidad hacia la esfera comunitaria y domiciliaria anunciado por las políticas y los planes de salud de las diferentes autonomías del Estado español ${ }^{(10-12.14)}$, giro que, a su vez, atribuye un creciente protagonismo a la persona cuidadora informal en los procesos de salud y cuidado ${ }^{(15-17)}$.

Una constante en las diferentes estrategias de mejora de la atención comunitaria y de los planes de salud consultados, así como de los artículos analizados, es la afirmación de que la enfermera tiene la responsabilidad de incorporar a la persona cuidadora como usuaria con derecho a mantener su salud ${ }^{(18)}$.

La mayoría de los artículos analizados comparten, implícita o explícitamente, una visión más utilitarista de la persona cuidadora, la cual es construida, presentada y tratada como un recurso, cuya salud es importante tener en cuenta en aras de garantizar que continúe siendo capaz de cuidar ${ }^{(19)}$. En definitiva, se detecta una tensión latente entre un abordaje preventivo a esta figura como futura paciente, por un lado, y un interés a menudo secundario por parte de la enfermería hacia ella y solo en tanto que recurso o colaboradora.

El análisis de las publicaciones indica que la enfermería no se plantea de manera comprehensiva su contribución específica en el marco de los nuevos paradigmas de cronicidad ni su posicionamiento sobre los impactos estructurales que éstos tienen sobre el cuidado informal $\left.\right|^{(9,20)}$. Ello genera un trasvase de la responsabilidad hacia el cuidado por parte de las administraciones públicas hacia las familias ${ }^{(14)}$.

Para concluir y respondiendo a las preguntas planteadas:

Las publicaciones se centran en estudios para conocer el perfil del cuidador y cómo planificar las intervenciones recomendando herramientas de valoración. Los estudios sobre cuidados informales, que tienen un gran impacto social en la emergente situación sanitaria, solo representan un $8,3 \%$ de las publicaciones en revistas de enfermería.

Los cambios reflejados en el abordaje de la dependencia son un retorno al escenario anterior a la LAPAD, volviendo la responsabilidad del cuidado a la familia en el entorno del propio hogar, siendo esta una de las tantas consecuencias de la crisis económica y los recortes en sanidad como apunta Rodríguez Cabrero ${ }^{(5)}$.

Los cambios en las competencias enfermeras están dirigidos a la atención comunitaria y domiciliaria teniendo en cuenta a la persona cuidada y a la persona cuidadora, pero enseñando y empoderando a éstas sobre la enfermedad y el autocuidado $^{(10,21,22)}$.

Las publicaciones no analizan las desigualdades socioeconómicas existentes en el cuidado, algunas mencionan sus impactos económicos o laborales sobre la persona cuidadora, sin problematizar que no todas las familias están en las mismas condiciones para contrarrestarlos y/o buscar alternativas. Las desigualdades sociales previas a la experiencia de cuidado promueven a su vez desigualdades adicionales de salud, de tiempo libre y, entre otras, laborales, entre las familias ${ }^{(23)}$. 
Si desde la enfermería se busca adquirir una visión compleja de los cuidados para poder dar respuestas efectivas, será necesario incorporar una atención específica a las desigualdades sociales presentes en ellos. Sobre todo en tiempos de crisis, deviene imprescindible considerar estos aspectos para sensibilizar la tarea enfermera a las realidades de las personas, tanto cuidadas como cuidadoras, tal como exponen Úbeda \& Roca ${ }^{(21)}$.

\section{CONCLUSIONES}

Las publicaciones enfermeras se centran en estudios para conocer el perfil del cuidador y cómo planificar las intervenciones recomendando herramientas de valoración.

Se identifica con acierto la centralidad del cuidado informal en los nuevos escenarios y paradigmas de cronicidad y, reflejan el giro asistencial hacia el domicilio y la familia. Se considera a la familia como la responsable de los cuidados siendo la mujer la principal proveedora de éstos.

No problematizan, sin embargo, el actual trasvase de responsabilidades hacia el cuidado desde las administraciones públicas hacia el ámbito familiar, ni analizan en profundidad las desigualdades socioeconómicas y de género reinantes en el actual escenario de cuidados.

El abordaje a estos dos elementos puede contribuir a abrir nuevas líneas de investigación e intervención en el campo de la enfermería.

\section{REFERENCIAS}

1. García-Calvente, MM. Cuidados de salud, género y desigualdad. Comunidad. 2012; 5: 3-4.

2. Rogero-García J. Distribución en España del cuidado formal e informal a las personas de 65 y más años en situación de dependencia. Rev Esp Salud Púb. 2009; 83:393-405.

3. García-Calvente, MM., Mateo-Rodríguez, I., Maroto-Navarro, G. El impacto de cuidar en la salud y la calidad de vida de las mujeres. Gaceta Sanitaria 2004; 18(II): 83-92.

4. Oliva J, Vilaplana C, Osuna R. El valor social de los cuidados informales provistos a personas mayores en situación de dependencia en España. Gaceta Sanitaria. 2011; 25(S): 108-114.

5. Rodríguez Cabrero G. Políticas sociales de atención a la dependencia en los Regímenes de Bienestar de la Unión Europea. Cuadernos de Relaciones Laborales [Internet]. 2011 [consultado el 15/10/2016]; 29(1): 13-42. Disponible en: http://revistas.ucm.es/index.php/CRLA/article/view/36184/35063

6. Calvente M, Del Río M, Marcos JM. Desigualdades de género en el deterioro de la salud como consecuencia del cuidado informal en España. Gaceta Sanitaria. 2011; 25: 100-107.

7. Segura, N., Gómez, R., López, R., Gil, E., Saiz, C., Cordero, J. El anciano dependiente y el desgaste físico y psíquico de su cuidador. Revista Española de Geriatría y Gerontología 2006; 41(I), 15-20. 
8. De Lorenzo R, Rivero A. La futura Ley de Dependencia como pilar fundamental de la protección social en España. Revista Española del tercer sector. 2006; (3): 4980.

9. Contel JC, Muntané B, Camp L. La atención al paciente crónico en situación de complejidad: el reto de construir un escenario de atención integrada. Atención Primaria. 2012; 44(2): 107-113.

10. Ferrer C, Orozco D, Román P. Estrategia para el Abordaje de la Cronicidad en el Sistema Nacional de Salud [Internet]. Madrid: Ministerio de Sanidad, Servicios Sociales e Igualdad; 2012 [consultado el 20/06/2016]. Disponible en: http://www.msssi.gob.es/organizacion/sns/planCalidadSNS/pdf/ESTRATEGIA ABORDAJE CRONICIDAD.pdf

11. Conselleria de Salut. Plan de atención a pacientes con enfermedades crónicas de la Comunidad Valenciana [Internet]. Generalitat Valenciana; 2012 [consultado el 25/09/2016]. Disponible en: http://iv.congresocronicos.org/documentos/plan-deatencion-pacientes-cronicos-valencia.pdf

12. Departamento de Sanidad y Consumo. Estrategia para afrontar el reto de la cronicidad en Euskadi [Internet]. Vitoria: Gobierno Vasco; 2010 [consultado el 24/10/2016]. Disponible en: https://www.osakidetza.euskadi.eus/r85skorga01/es/contenidos/informacion/estrategia cronicidad/es cronicos/estrategia croni cidad.html

13. Valderas JM, Starfield B, Sibbald B, Salisbury C, Roland M. Defining comorbidity: Implications for under standing health and health services. Ann Fam Med. 2009;7:357-63.

14. Departament de Salut. Pla de Salut de Catalunya 2011-2015 [Internet]. Generalitat de Catalunya; 2011 [consultado el 15/10/2016]. Disponible en: http://salutweb.gencat.cat/ca/el departament/pla de salut 2011 2015/

15. Rico-Blázquez M, Gómez SS, Gallego CF. El cuidado como elemento transversal en la atención a pacientes crónicos complejos. Enfermería Clínica. 2014; 24(1): 44-50.

16. Sánchez-Martín I. Cronicidad y complejidad: nuevos roles en Enfermería. Enfermeras de Práctica Avanzada y paciente crónico. Enfermería Clínica. 2014; 4(1): 79-89.

17. Rodríguez BE. Políticas de dependencia: consecuencias para las familias. Revista ROL de enfermería. 2008; 31(5), 22-32.

18. Escuredo-Rodríguez B. El discurso de las enfermeras ante el cuidado de las personas mayores dependientes y sus cuidadores familiares. Índex de Enfermería. 2006; 15(52-53), 45-48.

19. IMSERSO. Cuidados a las Personas Mayores en los Hogares Españoles. El entorno familiar [Internet]. Madrid: Ministerio de Trabajo y Asuntos Sociales. Secretaría de Estado de Servicios Sociales, familias y discapacidad; 2005 [consultado el 30/11/2016]. Disponible en: http://www.dependencia.imserso.es/InterPresent1/groups/imserso/documents/binario/c uidadosppmmhogares.pdf

20. Departament de Salut. Programa de prevenció i atenció a la cronicitat. Document conceptual. Versió 5.0 del 28.02.2012 [Internet]. Generalitat de Catalunya; 2012 [consultado el 05/11/2016). Disponible en: http://salutweb.gencat.cat/web/.content/home/ambits tematics/linies dactuacio/model assistencial/atencio al malalt cronic/documents/arxius/562conceptual.pdf

21. Úbeda I, Roca M. Los cuidados familiares y las políticas públicas. Nursing. 2008; 26(9): 56-58. 
22. Germán Bes C, Hueso Navarro F, Huércanos Esparza, I. El cuidado en peligro en la sociedad global. Enfermería Global [Internet]. 2011[consultado el 10/05/2016]; 10(23): 221-232. Disponible en: http://revistas.um.es/eglobal/article/view/125491

23. Delicado MV. Familia y cuidados de salud: Calidad de vida en cuidadores y repercusiones sociofamiliares de la dependencia [tesis doctoral]. Universidad de Alicante; 2003 [consultado el 12/11/2016]. Disponible en: http://hdl.handle.net/10045/3410 